\title{
Expert System of Fault Diagnosis of Dry-mixed Mortar Fieldbus Control System
}

\author{
Huan Zhang \\ Jiangsu Key Laboratory of Large Engineering Equipment \\ Detection and Control \\ Xuzhou Institute of Technology \\ Xuzhou, P. R. China \\ huan0804@163.com
}

\author{
Shuqi Shang, Dongwei Wang, Jiasheng Wang, \\ Ranbing Yang \\ College of Mechanical and Electrical Engineering \\ Qingdao Agricultural University \\ Qingdao, P. R. China \\ sqingnong@126.com, \{w88030661, \\ jiasheng0813,yangranbing\}@163.com
}

\begin{abstract}
A design and implementation of expert system of fault diagnosis of dry-mixed mortar based on Profibus fieldbus and large-scale PLC is proposed in this paper. Firstly, the control principle and algorithm, hardware choice and integration of the control system are introduced. Secondly, by analyzing the composition, structure and performance of drymixed mortar control system, the types, range, and causes of this control system were investigated. Finally, after comparing the fault diagnosis of traditional theories and modern ones, the expert system of fault diagnosis of this control system using Fault Tree Analysis was established.
\end{abstract}

Keywords-Dry-mixed mortar; filedbus control system; expert system; fault diagnosis; Fault Tree Analysis

\section{INTRODUCTION}

In recent years dry-mixed mortar, as a novel, green, environmental protection, energy-saving building material, is being popularized forcibly and widely in China. The research, development and practice application of this environmental protection material have been deeply proceed in the construction line. After analyzing the technology process and control demands of mortar production, a design of control system for dry-mixed mortar production line was proposed firstly in this paper, which include control system configuration, control strategy and so on. In the dry-mixed control system, the main control scheme we chosen is listed below: PLC as main controller and master station meanwhile, Profibus field-bus as data communication channel, and inverter, smart weighting instrument and other devices with field-bus interface as slave station. Via Profibus all electrical devices can be bidirectional interconnected as a whole and thus high speed data communication can be realized also. Advanced friendly Human-Machine Interface, like IPC (Industrial Personal Computer) or touch screen, were selected as host computer interface, which can perfectly realize the important data and recipe editing, download, the key data in the PLC upload, important process parameters displaying, key parameter setting, process monitoring and so on. At the same time, several intelligent algorithms, like advanced PID adjust and Bang-Bang controls were introduced, so the precision weighing of all kinds of raw material has been realized. By using large-scale PLC and smart weighing instrumentation, the motions of electric motor actuators or hydraulic cylinder actuators were run well, all technique process, like hoist, well-distribution by vibration, mixing, unloading, etc can be executed in time and in place. Though the actual running of the control system it has been proved that the fully integrated automation production of dry-mixed mortar can be thoroughly achieved.

Afterwards as the background of filedbus control system of dry-mixed mortar and according to system structure and performance of it, the types, range, and causes of the control system were studied. On the basis of studying the traditional theories and modern ones, the fault diagnosis model of the system by using Fault Tree Analysis was established. The supervision and control station with on the spot fault diagnosis function was built by using the strong diagnosis function and ability of S7-300 PLC and IPC, the high speed, bidirectional digital communication of PROFIBUS and selfdiagnosis of some intelligent actuator and driver. Finally, Expert system of fault diagnosis of the control system with some intelligence using expert system with FTA was implemented, and the software and hardware design of was completed, which include knowledge base, rule base, database and etc. The cooperative remote diagnosis mechanism between the enterprise fault diagnosis Center, remote fault diagnosis Center and diagonsis expert in the Internet by using Web technique, distributed technique were discussed as well.

\section{CONTROL DEMANDS AND PROCESS FLOW OVERVIW}

Before the design scheme of the control system for drymixed mortar are introduced, the process overview and control demands are presented briefly first.

\section{A. Control Demands}

On the basis of analyzing technologies process and work principle in the dry-mixed mortar production line, the main control demands of the electro mechanics system are enumerated as follows:

(a) The system should have manual control mode and automatic control one, and manual one mainly use in system 
maintenance or software debugging. In the normal production process, the general auto one is preferred. Therefore, the manual/automatic mode selection should be set, the corresponding button and set the indicator for each button, so that when the system is not working properly it is convenient to facilitate troubleshooting;

(b) Weighing instrumentation on the field can complete all kinds of ingredients materials weighing and measurement. Based on weighing signals and production formula, produce motions of screw conveyor, valves and other equipment are produced. Ingredients can dynamically detect and deal with problems that may occur during, communication between the controller and weighing meter can be done thru bi-directional, high-speed serial port with real-time;

(c) Switch logic controls of the external actuator such as the feeding of vibration, mixing and discharging are expected;

(d) Fault diagnosis and alarming, fault handling and alarm reset of control system;

(e) Human Machine Interface (HMI) should be beautiful and friendly. Through the HMI the recipe data editing, download of the key process parameters displaying, historical data and real time data curve depicting, at the same time Human Machine Interface can be communicated with main controller via serial communication.

\section{B. Process Flow}

Generally speaking, gel materials, aggregates and various additives and other components jointly constitute dry-mixed mortar. The function of gel is to glue aggregate and other particles together and bonded them firmly at the base layer, and which are mixed together with cement, gypsum and hydrated lime and so on in the mortar. The aggregate in drymixed mortar are usually selected yellow sand or artificial sand in some particle size according to certain grading standards. In common sense, dry-mixed mortar production line consists of material storage, weighing, feeding, vibration evenly, mixing and unloading, and some other production processes. During the dry-mixed mortar production, the main ingredient such as cement, fly ash and yellow sand and so on, and various additives are placed in their respective storage silos. In the production line the silo at the bottom and material distribution and weighing parts are connected to the upper parts of the production line by lifting and feeding devices. After distribution and weighing of the ingredients, raw materials in the scales cabin are conveyed with a screw conveyor into the top of the hopper. When the conveyance is completed, under the action of gravity the raw material are directly dropped down into the mixer. After the set time of mixing, the product is going into the discharge hopper, the end product of dry-mixed mortar in the discharge hopper can enter the packaging system finally.

\section{SYSTEM HARDWARE SELECTION AND INTEGRATION}

From the control demands mentioned above, the controller of the control system can process signals both digital and analog, having the ability of loop adjust of analog quantity. At the meantime the motors could run in the working condition of variable frequency, so the hardware selection of the control system for dry-mixed mortar production should be made deliberately.

\section{A. Main Controller}

As experienced electrical engineers known, PLC/PC (Program Controller) is a kind of popular industrial computer, by using it we can not only accomplish logic control, but also complete many advanced functions, such as analog loop adjust, and motion control, etc. According to the component amounts of input and output and the needs of control system, the large-scale PLC of S7-300 series is selected, which can doing analog adjust by using advanced instruction like PID instruction.

Because of the sampling and driving of the analog signal necessarily, CPU and digital input/output modules should be used together with analog input/output function module like SM331 and SM332 or some counterpart. On one hand, SM331 adopted is an analog input module having 8 channels with 13 bit high resolution, which could receive $0 \mathrm{~V} \sim+10 \mathrm{~V}$ voltage signal, $0 \sim 20 \mathrm{~mA}$ or $4 \sim 20 \mathrm{~mA}$ current signal. On the other hand, SM332 chosen could send standard voltage signal and/or current signal, having 4 channels with 15 bit high resolution also. It is something to be mentioned here, the wiring form of current input/output $(0 \sim 20 \mathrm{~mA})$ must be adopted in order to avoiding the strong electromagnetism disturbance in the working field.

\section{B. Speed Regulating Devices}

At present, inverter, as an import converter, can convert power frequency into continual frequency power. Thus, energy saving, cost consuming and noise reduction can be easily got by this equipment.

In this control system inverter of MM420 series of SIEMENS Corporation were elaborately chosen, which has many advantages, such as Vector Control (VC) and advanced applying macro and so on. Its main good points and characteristics are illustrated as follows: it can acquire maximum starting torque by using direct excitation; it can be applied to multiple driving systems by using master-slave function; input and output programmable function; high precision of speed regulating, perfect safeguard and alarming steps. Given these highlights of this inverter, pumps driving of stepless speed regulating can be easily obtained. There are many applying macro in MM420 series, but we should only choose corresponding macro here as we need.

\section{Monitor \& Control Interface}

IPC (Industrial Personal Computer) has strong reliability, compatibility, and extensibility, which can connect PLC with its serial port conveniently. In the hardware configuration IPC 547D series of SIMENS is chosen as HMI for the control system.

MCGS (Monitor Control Generated System), fashionable home-made industrial configuration software, is running on the SIEMENS IPC. With this HMI, the visualization of 
Monitor and Control is realized intuitively vividly and friendly.

With the sensor/transducer, analog input/output modules, S7-300 PLC and actuators, e.g. inverter and motor-driven valve, the loop adjust of steam pressure can be precisely attained, and temperature of all measure points could be detected also. The overall hardware configuration of the control system for dry-mixed mortar, please see figure 1 .

\section{INPLEMETATION OF EXPERT SYSTEM OF FAULT DIAGNOSIS OF THE CONTROL SYSTEM}

\section{A. Fault Tree Analysis and Expert Systemof Fault Diagnosis}

Fault tree analysis is a graphical method that applies logic reasoning to fault events under certain conditions. The aim of it is to identify the basic fault event, determine the cause of the failure, impact and probability of occurrence. The most undesirable events in the system is taken as the top event of fault tree, finding the basic reasons and the accidents that lead to the top event of all possible direct factors from top to bottom, and listing the logical relationship between each other with symbolic logic rules. Thus by in depth analysis gradually, the bottom events of the fault tree can be traced finally.

Fault diagnosis technology in the process of development and in different application fields, a variety of fault diagnosis methods combined with different subject are come from. Generally speaking, existing fault diagnosis methods can be divided into two categories that is the traditional method of fault diagnosis and approach based on artificial intelligence, e.g. expert system and fuzzy logic and etc. The traditional fault diagnosis methods can be divided into methods based on signal processing and the one based on analytical models. Each type of diagnostic methods includes a number of specific diagnostic methods. In the state of art modern fault diagnosis based on artificial intelligent is showing superiority increasingly. Because of the construction difficulty of diagnosis model, the expert system of fault diagnosis is proposed in this work.

In the expert system of fault diagnosis knowledge is collected together by the authoritative knowledge and data according to a certain form. But where are these authoritative knowledge and data come from? Man may very naturally think this is from experts, and many books about artificial intelligent and expert system introduce so just like that. It is worth mentioning that R. T. Hartley compared the knowledge from experts and the actual operator (or practitioner) in the relating field in the view of knowledge base, and then discussed the acquired knowledge in detail. He states that the required types of knowledge depend on the nature of tasks in the system belonging to the special field. If an expert system of fault diagnosis needs some fragmentary knowledge bind together to built knowledge base, owing to independence of the work of operators and different experiences of them, they can provide more valuable knowledge than one from experts, and which is additive as well. Therefore, the knowledge adopting here is derived from various sources, such as the experts, the engineer, the operators, and so on.

\section{B. Implemetation of Fault Diagnosis Using Fault Tree}

The fault types in the dry-mixed mortar production system are mainly consist of severe fault (the fault can cause casualty of men and equipments), machinery and hydraulic fault, electrical fault and etc. In view of specialty field we intensively investigated the electrical fault here.

Due to the expert system of fault diagnosis of the control system based on fault tree analysis, the establishment of the knowledge base is set up according to the fault tree principle. System failure in the fault diagnosis system is considered as top event - the root node of fault tree, and the direct reasons for system failure are viewed as the bottom events (intermediate events) - branch nodes of the tree, the logic symbol are used to connect them to the top event as well. Downwards progressively by analyzing intermediate events to produce branch nodes of fault tree until the basic event the leaf nodes were all produced at last. The fault diagnosis system using fault tree analysis is shown as Figure 2, taken electrical fault in the system as illustrated.

\section{CONCLUSION}

This production line of dry-mixed control system based on PLC, field-bus and industrial Ethernet is adopted totally distributed control architecture, realizing the industrial production process of fully distributed control and centralized management. After analyzing the composition, structure of dry-mixed mortar control system, the types and causes of this control system were studied in depth. Finally, the expert system of fault diagnosis of this control system using Fault Tree Analysis was established. It has been verified that this expert system of fault diagnosis is practical, available, and reliable in the actual run.

\section{ACKNOWLEDGMENT}

This paper is under the help of Associate Professor Hao $\mathrm{Li}$ of College of Automation, Wuhan University of Technology, and is financially supported by open project of Jiangsu Key Laboratory of Large Engineering Equipment Detection and Control (Project No. JSKLEDC201204).

\section{REFERENCES}

[1] H. Zhang, S. D. Li, The Principle of PLC with its Application to Process Control. Beijing: China Power Press, 2008.

[2] H. Zhang, The Shortcut to Control Systems of S7-300/400 Series PLC Using Diagrams. Beijing: China Machine Press, 2011.

[3] H. Zhang, "Design of Electrical Control System for Air Compressor Units," Process Automation Instrumentation. Shanghai, vol. 31, pp. 34-36, April 2010 (In Chinese).

[4] H. Zhang, "The Control System for Tread Production Line Based on CC-Link Fieldbus,” Rubber Industry. Beijing, vol. 57, pp. 359-361, June 2010 (In Chinese).

[5] L. A. Zadeh, Fuzzy Sets and their Applications. New York: Academic Press, 1975.

[6] G. R. Paul, "Modular field bus designs and applications," Assembly Automation. Bradford, vol. 20, pp. 40-46, January 2000.

[7] Information on http:// www.qdbst.net 


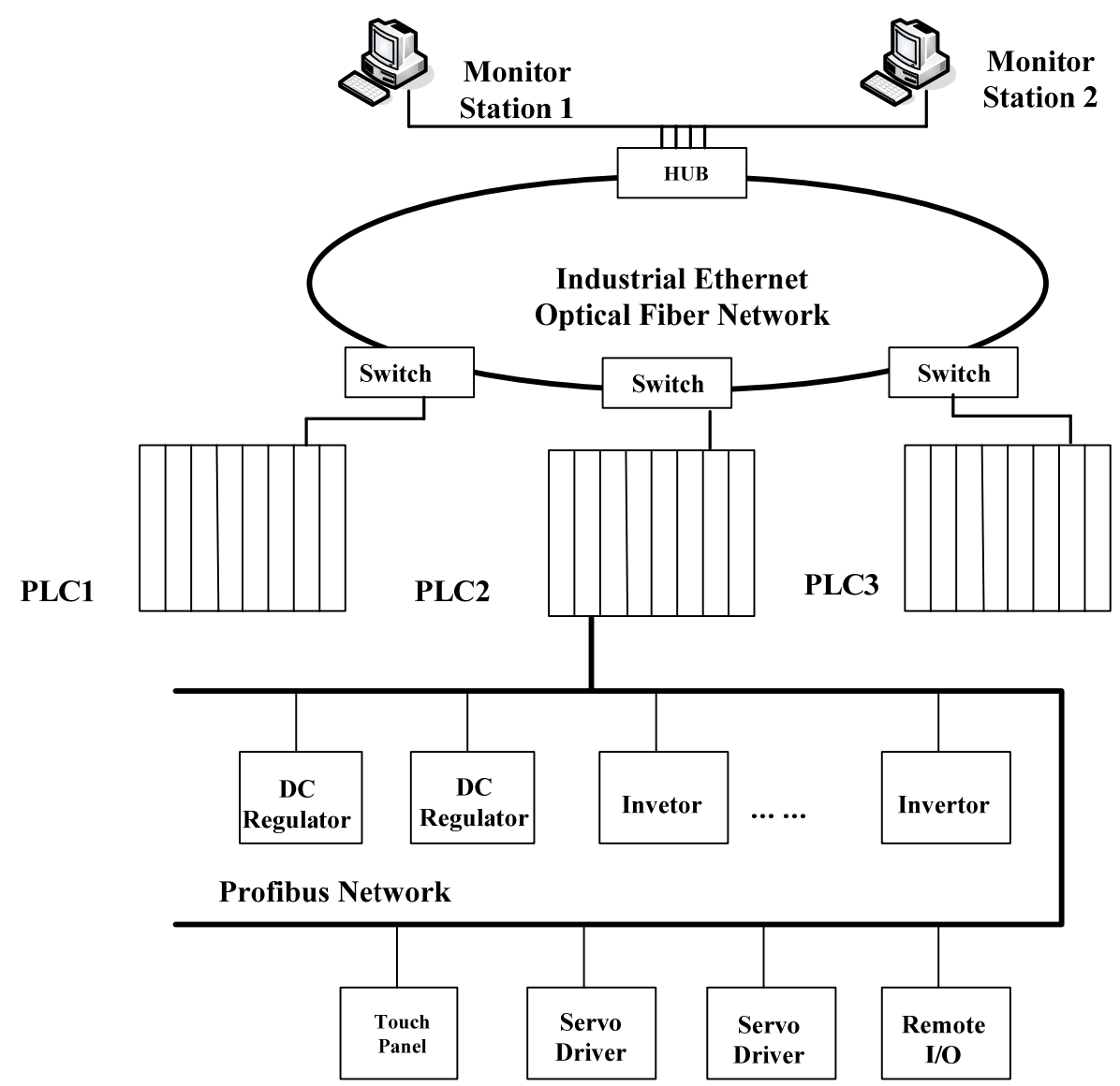

Figure 1. Overall hardware configuration of the control system for dry-mixed motar control system. 


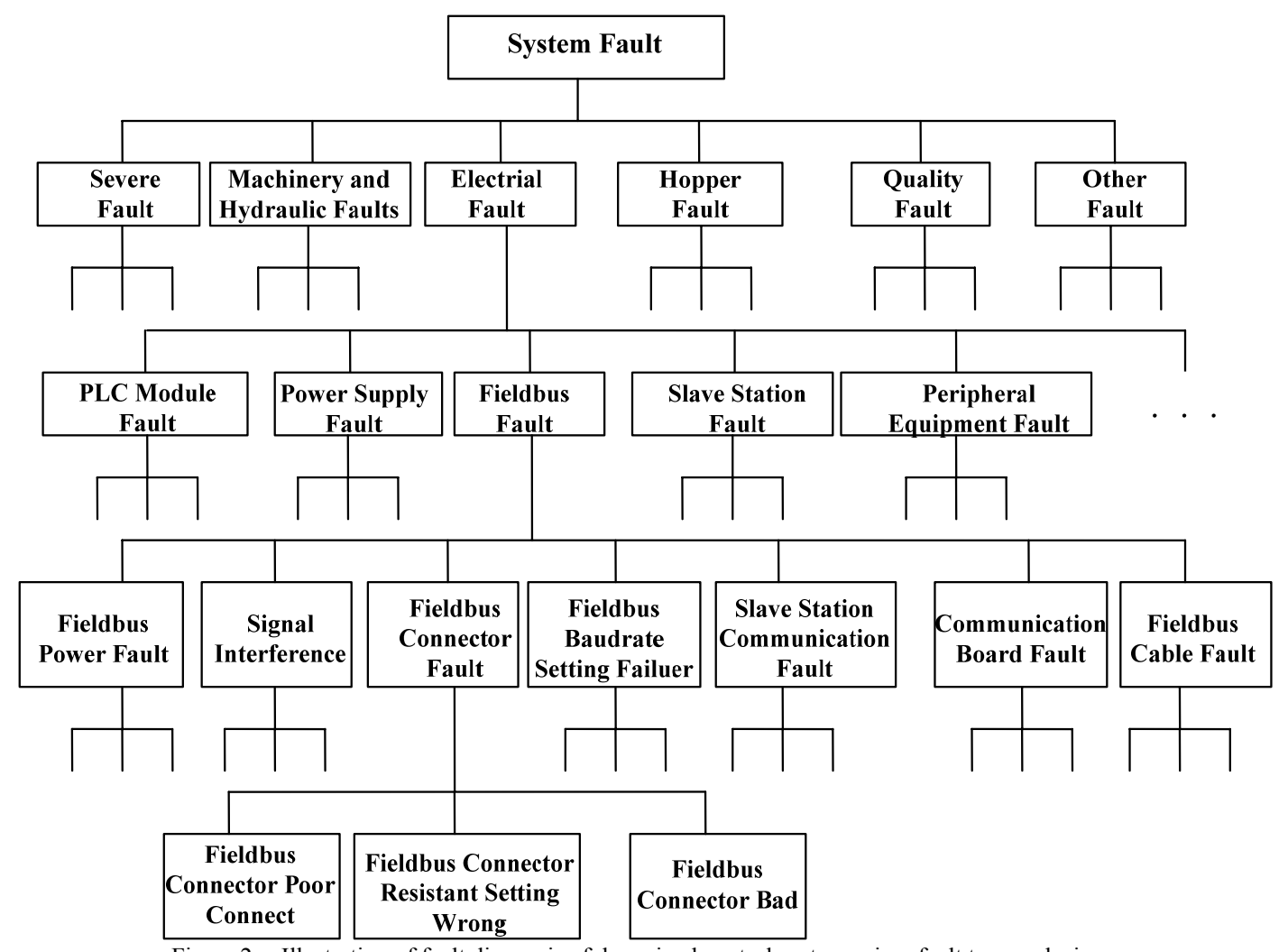

Figure 2. Illustration of fault diagnosis of dry-mixed control system using fault tree analysis. 